\title{
Article \\ SousChef System for Personalized Meal Recommendations: A Validation Study
}

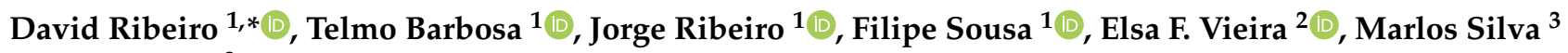 \\ and Ana Silva ${ }^{3}$ \\ 1 Fraunhofer Portugal AICOS, 4200-135 Porto, Portugal; telmo.barbosa@fraunhofer.pt (T.B.); \\ jorge.ribeiro@fraunhofer.pt (J.R.); filipe.sousa@fraunhofer.pt (F.S.) \\ 2 REQUIMTE/LAQV, Institute of Engineering of Porto, Polytechnic Institute of Porto, 4249-015 Porto, Portugal; \\ elsa.vieira@graq.isep.ipp.pt \\ 3 Sonae MC Serviços Partilhados, 4470-177 Maia, Portugal; mhsilva@sonaemc.com (M.S.); \\ amsilva@sonaemc.com (A.S.) \\ * Correspondence: david.ribeiro@fraunhofer.pt
}

check for updates

Citation: Ribeiro, D.; Barbosa, T.;

Ribeiro, J.; Sousa, F.; Vieira, E.F.; Silva, M.; Silva, A. SousChef System for Personalized Meal

Recommendations: A Validation

Study. Appl. Sci. 2022, 12, 702.

https://doi.org/10.3390/

app12020702

Academic Editor: Ángel

González-Prieto

Received: 10 December 2021

Accepted: 9 January 2022

Published: 11 January 2022

Publisher's Note: MDPI stays neutral with regard to jurisdictional claims in published maps and institutional affiliations.

Copyright: (C) 2022 by the authors. Licensee MDPI, Basel, Switzerland. This article is an open access article distributed under the terms and conditions of the Creative Commons Attribution (CC BY) license (https:// creativecommons.org/licenses/by/ $4.0 /)$.

\begin{abstract}
Nutrition is an essential part of our life. A healthy diet can help to prevent several chronic diseases like diabetes, obesity, cancer, and cardiovascular diseases, being influenced by social, cultural, and economic factors. Meal recommender systems are a trend to assist people in finding new recipes to cook and adopt healthier eating habits. However, food choice is complex and driven by multiple factors which need to be reflected in the personalization process of these systems to ensure their adoption. We present SousChef, a meal recommender system that can help to plan multiple meals considering an individual's food preferences, restrictions, and nutritional needs. Our approach uses recipes rather than individual food items, limiting recommendations to tasteful and culturally acceptable food combinations. Several experiments were performed to evaluate the system from different perspectives: nutritional, food preferences, and restrictions, and the recommendations' variability. Our results highlight the importance of using extensive and diverse content in recommendations to meet food preferences, restrictions, and nutritional needs of people with different characteristics.
\end{abstract}

Keywords: food recommender systems; health and safety; meal recommender systems; nutrition; optimization; recommender systems

\section{Introduction}

Nutrition plays a vital role in our health and well-being, and according to World Health Organization (WHO), a healthy diet can help prevent several chronic diseases, including diabetes, obesity, cancer, and cardiovascular diseases [1]. In most cultures, it is also a joyful experience and an essential part of social life. In the past, recommender systems have proven their potential to deal with information overload, especially in the retail domain. In the health domain, recommender systems help people find new recipes to cook or support them in adopting healthier eating habits to prevent health complications [2]. Some systems provide single meal recommendations that assist users in finding healthy meal ideas and new food variety.

Others [3,4] can help users manage diseases like diabetes by generating menus for larger periods, being able to consider daily nutritional requirements and the nutritional interaction between different meals.

However, there is still a need for systems to guide people on their food choices on a daily basis to help prevent diseases with a healthy diet. Moreover, food choice is complex, involving multiple factors. The validation of existing systems is often limited to nutritional aspects even though there are other important aspects for the acceptability of recommendations that should be considered. This work presents the validation of SousChef, 
a meal recommender system capable of creating personalized meal plans for multiple meals considering user preferences, restrictions, and nutritional needs to support the adoption of healthier eating habits. Since its initial development [5-7], its flexibility has been improved to adapt to users' eating habits, allowing them to personalize the number of meals. Unlike other approaches $[3,4]$, the recommendations of SousChef are not focused on managing specific diseases, they aim at providing daily support towards their prevention through contextual personalization. The recommendations use recipes rather than independent food items, which allows for a more tasteful and culturally acceptable experience.

SousChef can be easily extended with additional criteria and constraints to select meals considering new users or food information. For the validation, extensive experiments and simulations were performed to evaluate whether SousChef's recommendations are suitable considering different important criteria for food choice: nutrition, preferences and restrictions, and variability of the recommendations. Our experiments highlight the importance of the content used in the recommendations. Extensive and diverse content is necessary to be able to meet users' expectations.

The rest of this paper is organized as follows. Section 2 presents the related work. Section 3 describes the SousChef meal recommender system, namely how meal recommendations and the algorithm were modelled. Section 4 presents the validation of SousChef based on simulations, followed by Section 5 with the discussion of the achieved results. Section 6 draws the main conclusions and points out the future work.

\section{Related Work}

Meal recommender systems have the potential of improving health and quality of life by assisting health professionals and people in general planning meals to accomplish a balanced diet [2]. We have looked at the different approaches for creating decision support systems for personalized nutrition, and we have organized them based on how they support people in their lives: (1) Single Meal Recommender Systems, which offer isolated suggestions, and (2) Menu Generation Systems, which offer full-fledged plans for multiple meals, for a single day, or even for an entire week.

\subsection{Single Meal Recommender Systems}

Single meal recommendation systems can help people choose recipes by creating personalized recommendations based on food preferences, healthiness, or a list of available ingredients. Although they are not intended to give full dietary advice as menu generation systems, they can have a positive impact on eating decisions. For instance, a study [8] concluded that users tend to make healthier choices when a healthy tag is simply displayed together with the recommendations. These systems can also give support to dietitians' decision-making on which food is suitable for which patients. A previously developed system [9] explores how features such as age, gender, weight, disease, users' nutritional needs, and food nutritional content can be used to create food recommendations. Besides health-related matters, systems may also help users with their day-to-day decisions. The authors in [10] propose a solution to recommend recipes maximizing the number of ingredients from an input list. This allows users to find recipes that match their preferences or the ingredients they have available at home.

Recommender Systems from other domains create single recommendations based on users preferences, ranging from outfit recommendations [11] to e-commerce to increase sales and improve user experience [12]. Many factors can be taken into account to provide recommendations and increase accuracy, including sentimental analysis [13] or fashion stereotypes [14]. In the meal recommendation context, the parameters used to do the predictions can vary from looking at the ingredients of a recipe, the recipe's illustrative images [15], or food preferences using collaborative filtering [16].

Single meal recommender systems may be used for helping users make healthier choices or simply have recommendations based on their preferences or available ingredients. However, these fall short in providing users with balanced and diverse dietary advice. 


\subsection{Menu Generation Systems}

Unlike single meal recommender systems, menu generation systems create recommendations for multiple meals, comprising larger periods in the recommendations. Having extended control over the users' diet, these systems can help them follow a more balanced and diverse diet. Just like single meal recommender systems, these systems can also consider users' preferences and restrictions.

A system has been developed [17] that can create daily meal plans that includes the food preferred by the user, not consumed recently, and satisfies the daily nutritional requirements. It implements a pre-filtering stage that uses AHPSort as a multi-criteria decision analysis tool for filtering out foods that are not appropriate to user's characteristics and use features for each meal-such as previous frequency, previous last consumption, and preference value-to ensure the user's preferences and diversity are met on the recommendations. Another example [15] uses a neural network to predict users' preferences on recipes based on three key factors: the users' history, recipes' list of ingredients, and descriptive images.

Some systems in this category implement health-aware methods, providing a meal plan based on the user's disease needs/restrictions. In [4], an integer programming algorithm was implemented to create meal recommendations that meet the daily nutrient requirements for diabetic persons, minimizing its cost. The solution developed in [3] can recommend single, daily, or weekly meal plans, taking into account diabetics' concerns on the plan estimation but also patient's cultural, social, economic, and biological status, physical characteristics, and health concerns by using a Multi-Criteria Decision Making Approach (MCDM). Machine learning is also used in the development of meal recommender systems, as in [18] where a TensorFlow neural network model is used to classify recipe images to determine whether they are recommendable given the characteristics of a diabetic patient. There is a trend of these systems to support people with diabetes because a balanced diet has a positive impact on their well-being, but these systems can also help manage other diseases such as thyroid disorders. A support system has been implemented [19] using K-nearest neighbours and collaborative filtering models to suggest balanced diets for thyroid patients meeting their special nutrient requirements.

The approaches and the problems tackled by the different studies are diverse (Table 1) and use private or hard to replicate datasets, which makes results harder to compare. The validation of the approaches often focuses on the nutritional suitability of the recommendations, disregarding other important food choice criteria.

Table 1. Summary of the related work comparing the scope of meal recommendations, the disease they are focused in, the items used in recommendations, and the focus of their validation.

\begin{tabular}{|c|c|c|c|c|c|c|}
\hline & Single Meal & Daily Meal & Week Meal & Disease Focus & Items & Validation Focus \\
\hline [8] & $x$ & & & - & Recipes & Preferences \\
\hline [9] & $\mathrm{x}$ & & & Any & Food products & Classification problem (f1 measure) \\
\hline [10] & $x$ & & & - & Recipes & Processing time \\
\hline [15] & $x$ & & & - & Recipes & Preferences \\
\hline [16] & $\mathrm{x}$ & & & - & Recipes & Preferences \\
\hline [18] & $\mathrm{x}$ & & & Diabetes & Recipes & Nutritional requirements \\
\hline [19] & $\mathrm{x}$ & & & Thyroid & Recipes & $\begin{array}{l}\text { Nutritional requirements and } \\
\text { preferences }\end{array}$ \\
\hline [17] & & $x$ & & - & Food items & $\begin{array}{l}\text { Nutritional requirements, variability } \\
\text { and preferences }\end{array}$ \\
\hline [4] & & $x$ (2 days) & & Diabetes & Recipes & Nutritional requirements and cost \\
\hline [3] & & $x$ & $x$ & Diabetes & Recipes & Nutritional requirements \\
\hline
\end{tabular}


In this study, we present our approach for the validation of a meal recommender system capable of providing personalized recommendations for multiple meals, considering multiple objectives, and using recipes in the recommendations. We have conducted lab validation of the recommender system with synthetic user profiles which considered different features relevant to a healthy diet.

\section{SousChef Meal Recommender System}

The objective of the SousChef meal recommender system is to assist its users in planning their meals by allowing them to request personalized suggestions for multiple meals. Since its previous versions [5-7], where users were restricted to creating plans for an entire week and 6 meals per day, it has been improved to increase personalization by giving users more flexibility. Users may now get recommendations for the meals they usually have, and they can plan any subset of meals in the week. Users have a mobile application to interact with the system, which allows them to insert their anthropometric data and information regarding eating habits, food preferences, and dietary restrictions. They can use the application to plan their meals by receiving suggestions for any set of meals within a week, as well as alternatives to these suggestions. The recommendations are personalized using the user profile and food information.

\subsection{Problem Formulation}

Similar to other approaches $[3,4,17,20]$, we modelled meal recommendations as a multi-objective optimization problem. We consider each slot to be planned as a meal division $(M d)$, which is a part of a meal such as a lunch's starter, dinner's dessert, or fruit for a mid-afternoon snack. In this context, the slots are interdependent, as the choice for a given meal division often impacts the scores for another (e.g., lunch's main dish affects the choice for dinner's main dish). The scope to be planned (scope) is defined as a set of meal divisions (Figure 1). Recipes $\left(R_{c p}\right)$ are the candidates that need to be selected for each $M d$. These need to be inserted in the system beforehand and may be suitable for multiple $M d \mathrm{~s}$, meaning that a recipe for dinner's main dish may also be suitable for lunch's main dish. The meal planning context $\left(M_{p c}\right)$ puts together all the information available about the user-including their anthropometric data, nutritional requirements, meal history, food preferences, and restrictions-and about food, including nutritional and pricing information. Hard restriction functions may be defined that consider the meal planning context and allow filtering inadequate recipes by considering the $M_{p c}$. The scoring function to be optimized is defined by combining a set of heuristic functions $(H)$ reflecting different objectives. A variable number of heuristics may be defined as three types according to the scope they consider: single meal divisions $\left(H_{m d}\right)$, days $\left(H_{d}\right)$, or week $\left(H_{w}\right)$. A weight $w$ is also defined for each heuristic function to define its relative importance. Each function considers the meal planning context and the recipes of the scope to attribute a normalized score to each recipe: meal division heuristics consider a single recipe for a meal division, day heuristics consider all the recipes planned for a given day attributing a score to each of them, week heuristics consider the planning for the whole week and give a score to each of those recipes. The heuristic functions are combined according to Equation (1), consisting of the sum of the weighted average of the scores attributed by the heuristics of each scope. This ensures that, even though different weights may be attributed to each heuristic function, the different scopes have the same importance and are not influenced by the number and weight of the heuristics defined for each scope. The goal is to select a combination of recipes, one for each $M d$ in the selected scope, that maximizes the score considering the defined heuristics (Equation (1)). 


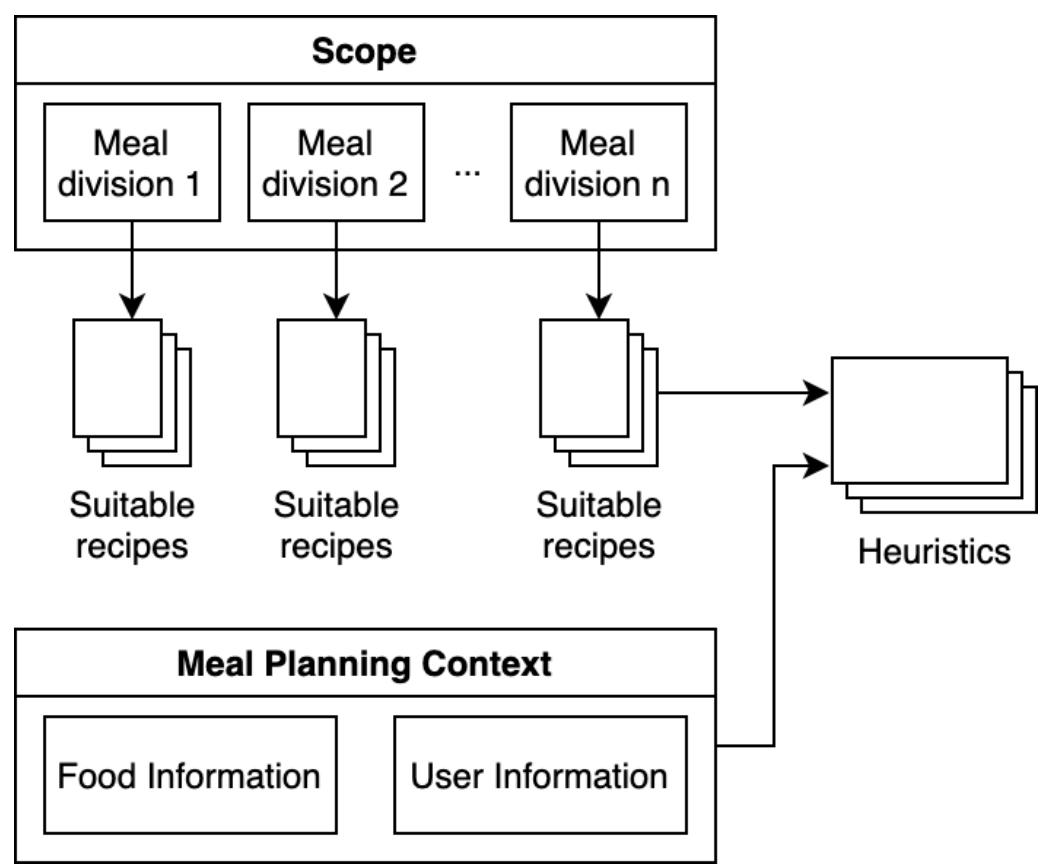

Figure 1. Overview of the concepts used in the problem formulation.

$$
\begin{gathered}
\operatorname{Maximize} \frac{\sum_{h_{m d} \in H_{m d}} w_{h_{m d}} * h_{m d}\left(M_{p c}, R_{c p}\right)}{\sum_{h_{m d} \in H_{m d}} w_{h_{m d}}}+ \\
\frac{\sum_{h_{d} \in H_{d}} w_{h_{d}} * h_{d}\left(M_{p c}, R_{c p}\right)}{\sum_{h_{d} \in H_{d}} w_{h_{d}}}+ \\
\frac{\sum_{h_{w} \in H_{w}} w_{h_{w}} * h_{w}\left(M_{p c}, R_{c p}\right)}{\sum_{h_{w} \in H_{w}} w_{h_{w}}}
\end{gathered}
$$

\subsection{Algorithm}

The meal recommender algorithm starts by compiling the meal planning context that refers to the user in question. The necessary information is cached whenever possible to improve performance. A greedy search approach (Figure 2) is then used to select the best combination of recipes, planning the meals one by one and beginning the exploration of the search space with the most promising solutions. For each meal division being planned, the list of suitable candidates is created and filtered using all defined restriction functions. The meal divisions are then planned one by one by evaluating the scoring function for each candidate. The candidates are then distributed into numberofcandidates / 5 bins using this score, and a roulette wheel selection from the top-performing bin is used to select the candidate and introduce variability in the resulting solutions. Since meals are planned one by one, the day and week heuristics are only included in the equation once all meals for their scope have been planned. An iterative cycle is performed after each day and week, and they are planned to allow these heuristics to improve the solution by changing already selected recipes. In each cycle, all meals that have been planned so far are evaluated by the heuristics, and the lowest scoring recipe is replaced with the best performing alternative. To reduce the time needed to create plans and avoid infinite loops, these cycles are ended after a predefined number of iterations or no improvements are achieved. The algorithm is also capable of generating alternative suggestions for a selected meal. In this case, the heuristics are evaluated on all alternative candidates and a list sorted by the score is returned. 


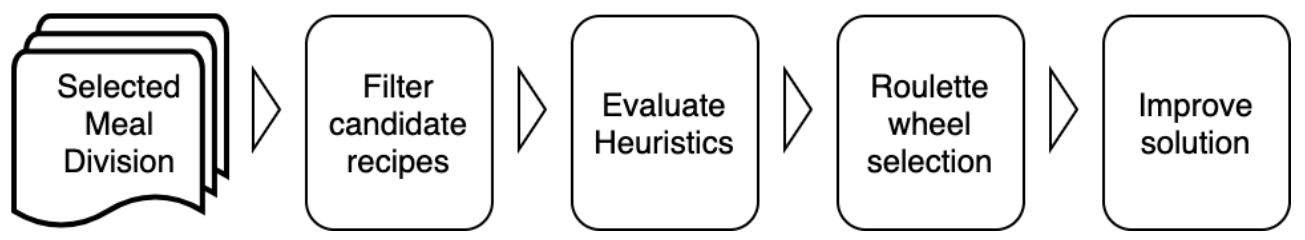

Figure 2. Overview of the SousChef planning algorithm.

\section{Validation Study}

Validation studies with end-users allow us to test the meal recommender system in a real-world environment. However, they can be expensive and take a very long time to carry out [21-23], thus they cannot be carried out very often and are not appropriate for all stages of development.

The proposed recommender system has been validated according to other approaches from recommender systems in the food and other domains. Since public datasets from this domain are not available, we performed a lab validation of the recommender system by considering multiple factors impacting food choice, which should be reflected in a system of this kind.

The recipes used in the recommendations have been compiled by a nutritionist for the different meal divisions, considering an average $2000 \mathrm{kcal}$ diet of the senior population [5-7]. They were based on the Portuguese food composition database compiled by INSA [24]. The recipes were created assuming 6 meals per day, including snacks. Each meal has been subdivided into multiple meal divisions according to Table 2, which also shows the number of recipes per meal division. For this purpose, each recipe contains a list of food composition database ingredients and respective quantities that allow the calculation of the nutritional value of that meal. Each ingredient in the database is also described using the LanguaL thesaurus [25] which enables, for instance, to know the type of ingredients in a recipe and consider this information in the heuristic functions.

Table 2. Number of recipes and unique ingredients per meal division.

\begin{tabular}{llcc}
\hline \multicolumn{2}{c}{ Meal Division } & Recipe Count & Unique Ingredient Count \\
\hline \multirow{3}{*}{ Breakfast } & Cereal & 118 & 34 \\
& Fruit & 30 & 30 \\
& Milk prod & 10 & 8 \\
\hline \multirow{2}{*}{ Morning Snack } & Fruit & 30 & 30 \\
& Main Dish & 126 & 38 \\
\hline \multirow{3}{*}{ Lunch } & Dessert & 37 & 37 \\
& Main Dish & 203 & 135 \\
\multirow{2}{*}{ Afternoon snack } & Soup & 24 & 34 \\
& Fruit & 30 & 30 \\
& Main Dish & 129 & 39 \\
\hline \multirow{2}{*}{ Dinner } & Dessert & 34 & 34 \\
& Main Dish & 198 & 133 \\
& Soup & 24 & 34 \\
\hline \multirow{2}{*}{ Supper } & Fruit & 27 & 27 \\
& Main Dish & 119 & 35 \\
\hline
\end{tabular}

The meal recommender system has been configured with heuristics to reflect users' food preferences, convenience (e.g., reduce the number of ingredients to facilitate shopping and planning the same soup in consecutive meals), nutritional needs (e.g., favour recipes closer to user energy and macronutrient needs), and variety (e.g., do not serve the same side dish twice in a row). Restrictions have also been defined to remove recipes with any ingredient specified by the user, filter some non-matching recipe combinations, and enforce 
food restrictions like lactose intolerance, vegetarian, and vegan diets. A nutritionist was involved to inform the development of the system and its heuristics.

A test population of 100 profiles has been randomly generated [17], consisting of their height $(158.35 \pm 8.37 \mathrm{~cm})$, weight $(74.24 \pm 12.86 \mathrm{~kg})$, birthdate (age $74.52 \pm 4.02$ years), gender (60\% female), number of meals per day (3: $30 \%, 4: 29 \%, 5: 18 \%$, or $6: 23 \%)$, activity level (all set to the lowest level in the scale), and random recipe ratings (10 positive and 10 negative). The distributions of height and weight parameters were approximated to the senior Portuguese population [26], the chosen activity level is the most common within this age group and reduces the number of confounding factors. The system allows users to configure the meals they usually have, and the number of meals is translated into meals according to the following priority: breakfast, lunch, dinner, afternoon snack, morning snack, and supper. The total energy requirement was estimated for each user [27], resulting in $1811.82 \pm 294.36 \mathrm{kcal}$. The energy is distributed across the macronutrients as $55 \%$ carbohydrates, $20 \%$ for protein, and 25\% for fat, following the 2015-2020 USA Dietary guideline [28]. A different distribution could be used or one could be specified for each individual.

The meal recommender was then used to generate plans for each profile for three consecutive weeks including all selected meals. Different metrics were then calculated using the generated plans to validate the system according to different topics presented next: nutrition, food preferences and restrictions, and variability.

\subsection{Nutrition}

Nutrition support is an important motivation for the development of meal recommender systems. The nutrition suitability of the recommendations is also frequently validated in other approaches in the literature $[3,4,17]$. Different factors need to be ensured for the continued use of the meal recommender system throughout multiple weeks. The most important factor is to meet the energy requirements and a healthy distribution of macronutrients. The Institute of Medicine (IOM) has developed a new approach for establishing recommended dietary allowances for macronutrients. The 2015-2020 Dietary Guideline recommends that carbohydrates comprise $45-65 \%$ of calories, fat $25-35 \%$ of calories, and protein 10-30\% of calories [28]. In this regard, we calculate different metrics to measure the nutritional suitability of the recommendations created by the system. The metrics we used were the average percentage deviation of energy and macronutrients between the meals planned for each day and the estimated daily nutritional requirements. The percentual deviations are $-5.86 \pm 11.33 \%$ for energy, $-8.2 \pm 13.87 \%$ for carbohydrates, $-8.49 \pm 12.36 \%$ for protein, and $1.34 \pm 12.48 \%$ for fat. The fact that the standard deviations are high compared to the mean values led to looking for an explanation for the deviation. While doing this, we found a significant correlation between the deviations and the number of meals, represented in Figure 3.

\subsection{Food Preferences and Restrictions}

Another important factor for the success of this kind of system is to meet user expectations regarding their personal food preferences and restrictions. To achieve sustained use and support meal planning on a daily basis, menu generation systems need to incorporate users' preferences in their recommendations. To measure this effect, we calculated the prevalence of recipes that were liked and disliked by users in the meal plans. The number of liked and disliked recipes in each week plan was correspondingly $6.75 \pm 5.27$ and $0.57 \pm 1.58$. Since users have different numbers of meals, and to facilitate comparison with different systems, we have also normalized this value dividing by the number of planned meal divisions for each week. The normalized value for the inclusion of liked and disliked recipes are correspondingly $8.26 \pm 6.05 \%$ and $0.7 \pm 1.97 \%$.

To validate the consideration of food restrictions, we need to ensure that food not meeting user restrictions is excluded from recommendations. This validation is simple in the case of our system, since food restrictions are manually mapped to the ingredients and 
therefore recipes that need to be excluded. Therefore, we have used unit tests to ensure correct behaviour and they were not included in user profiles to reduce entropy in the analysis of the remaining factors.

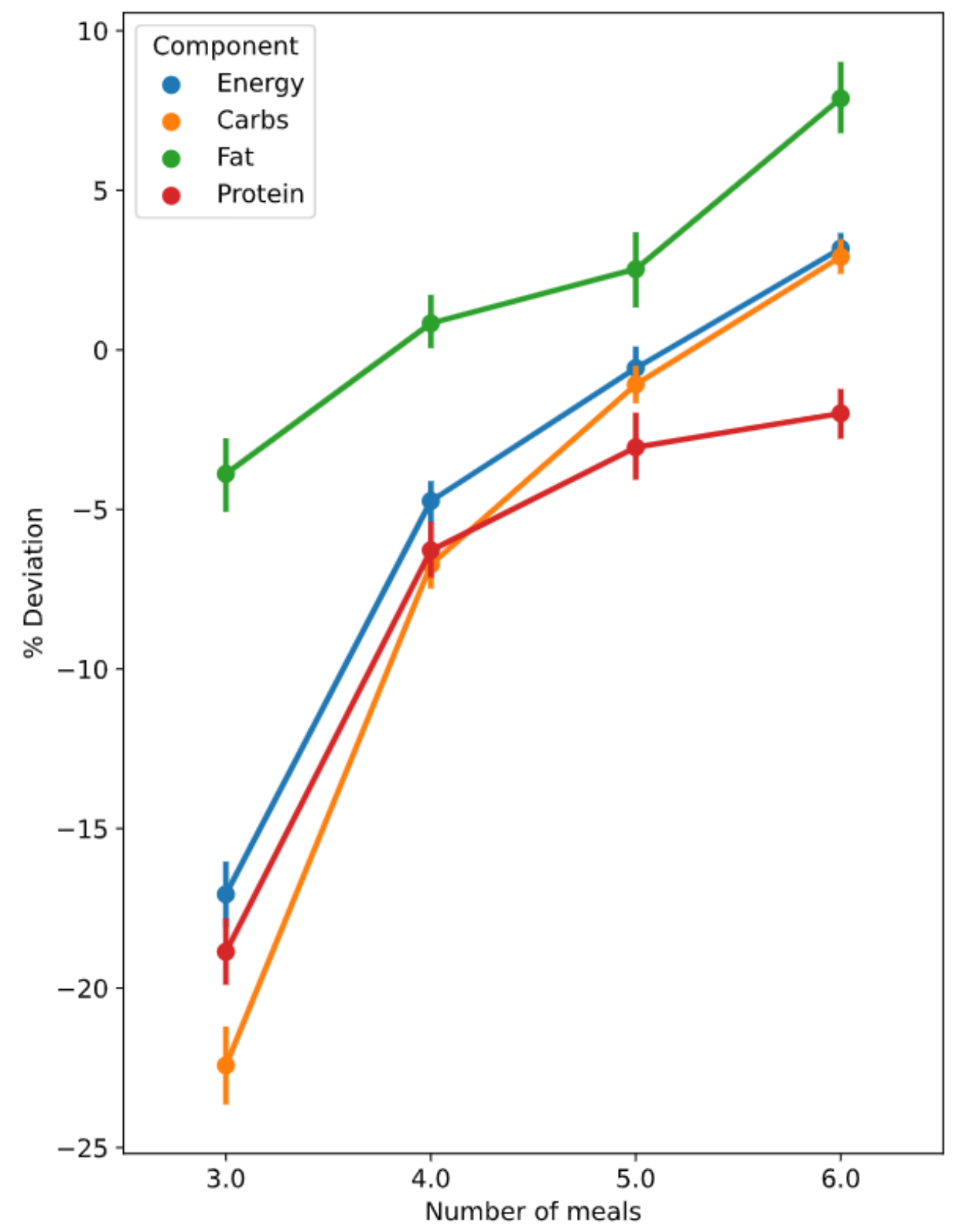

Figure 3. Error plot representing the daily percentual deviations of energy and macronutrients between needs and planned meals (Components) for each selected number of meals.

\subsection{Variability}

Finally, variability or novelty is both an important aspect of a healthy diet and a recommender system. To encourage the sustained use of a meal planning assistance tool, different meals should be recommended throughout time to maintain users' interest. To measure this effect, we have calculated the number of unique ingredients and recipes included in each week plan: $66.84 \pm 6.01$ ingredients and $41.35 \pm 4.62$ recipes. We have also normalized them by dividing by the number of meal divisions: $0.84 \pm 0.15$ for ingredients and $0.52 \pm 0.08$ for recipes. These are representative of the variability within a week plan, however, it is also important to ensure variability across different weeks. Therefore, we also calculated the number of overlapping ingredients and recipes between each consecutive week plans of each user: $146.97 \pm 21.36$ for ingredients and $71.53 \pm 15.17$ for recipes. Since 
users have different numbers of meals planned, these are the percentage of ingredients and recipes that overlap between two consequent weeks: $92.71 \pm 3.11 \%$ for ingredients and $87.31 \pm 4.62 \%$ for recipes.

Like [17], we analysed the frequency of repetitions. We also analysed their relation to the number of meals (Figure 4) and the number of recipes available for each meal division (Figure 5).

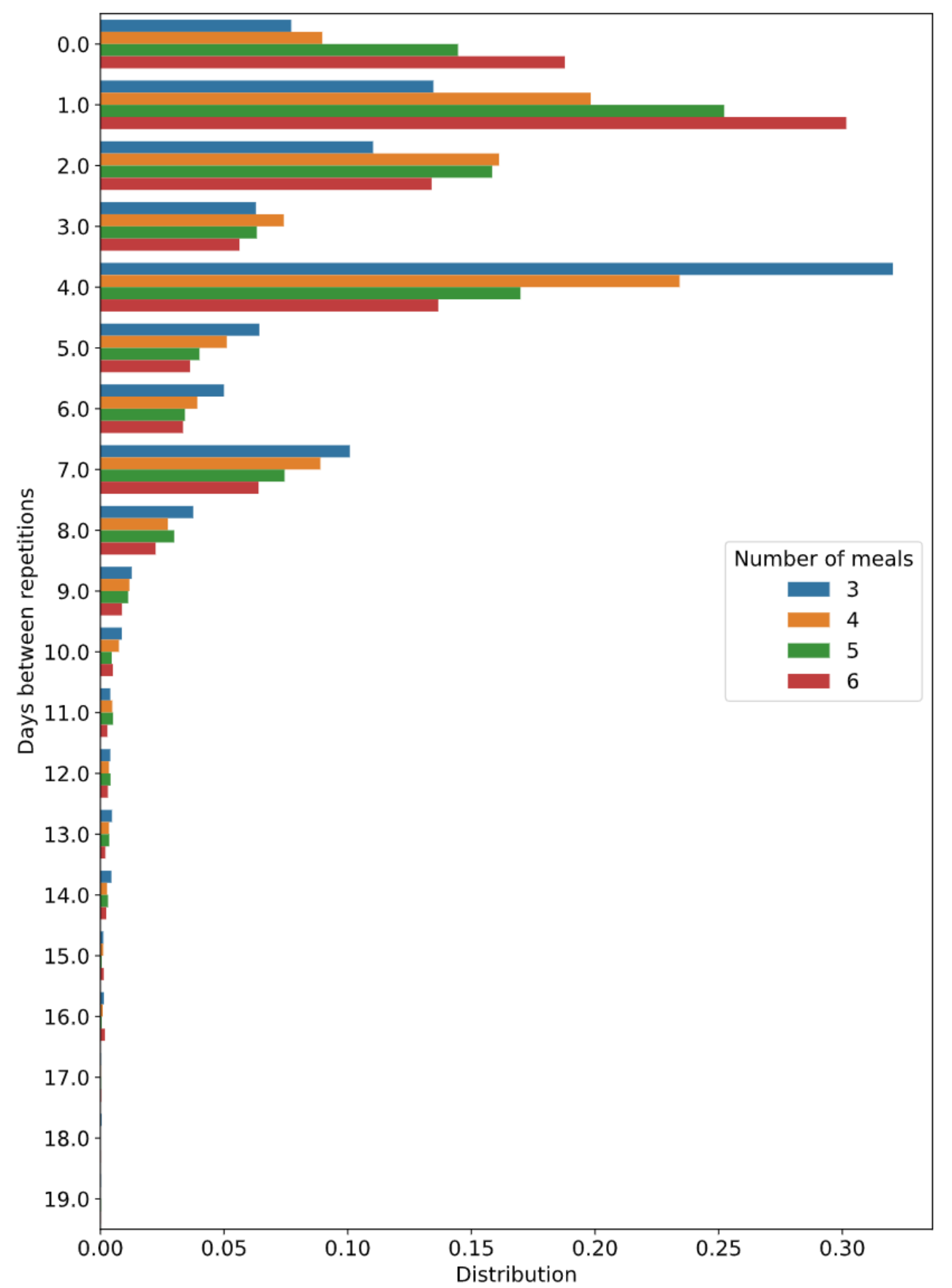

Figure 4. Distribution of the number of days between repetitions for the different numbers of selected meals. 


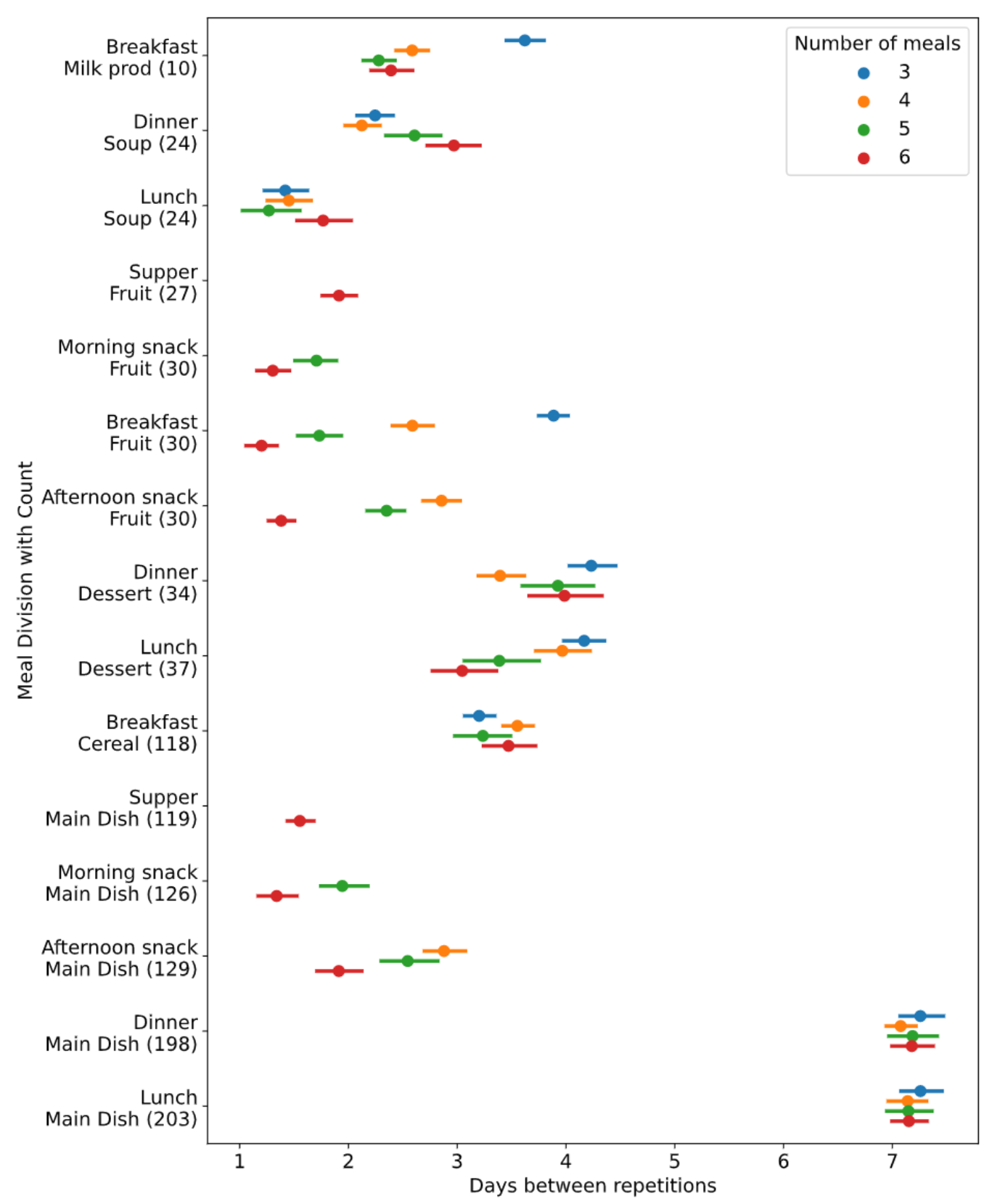

Figure 5. Plot of the number of days between repetitions in the meal plans and the different meal divisions sorted by the number of available recipes inside parenthesis.

\section{Discussion}

The meal recommender system has been tested using simulated user profiles from different perspectives. From a nutritional standpoint, the plans generated by the system were able to meet a low deviation from user needs when a high number of meals is selected. A lower number of meals requires concentrating the energy and macronutrient needs in a lower number of meal divisions. The recipes available in the system were created considering 6 meals, hence the higher deviations may be explained by the difference in energy content of the available recipes (which summarizes other macronutrients) and users' estimated nutritional needs for each meal division (Figure 6). This highlights the importance of the content used in the recommendations, besides the efficacy of the approach. The high-fat deviations are also a result of the recipes and the food available in the food composition database. 


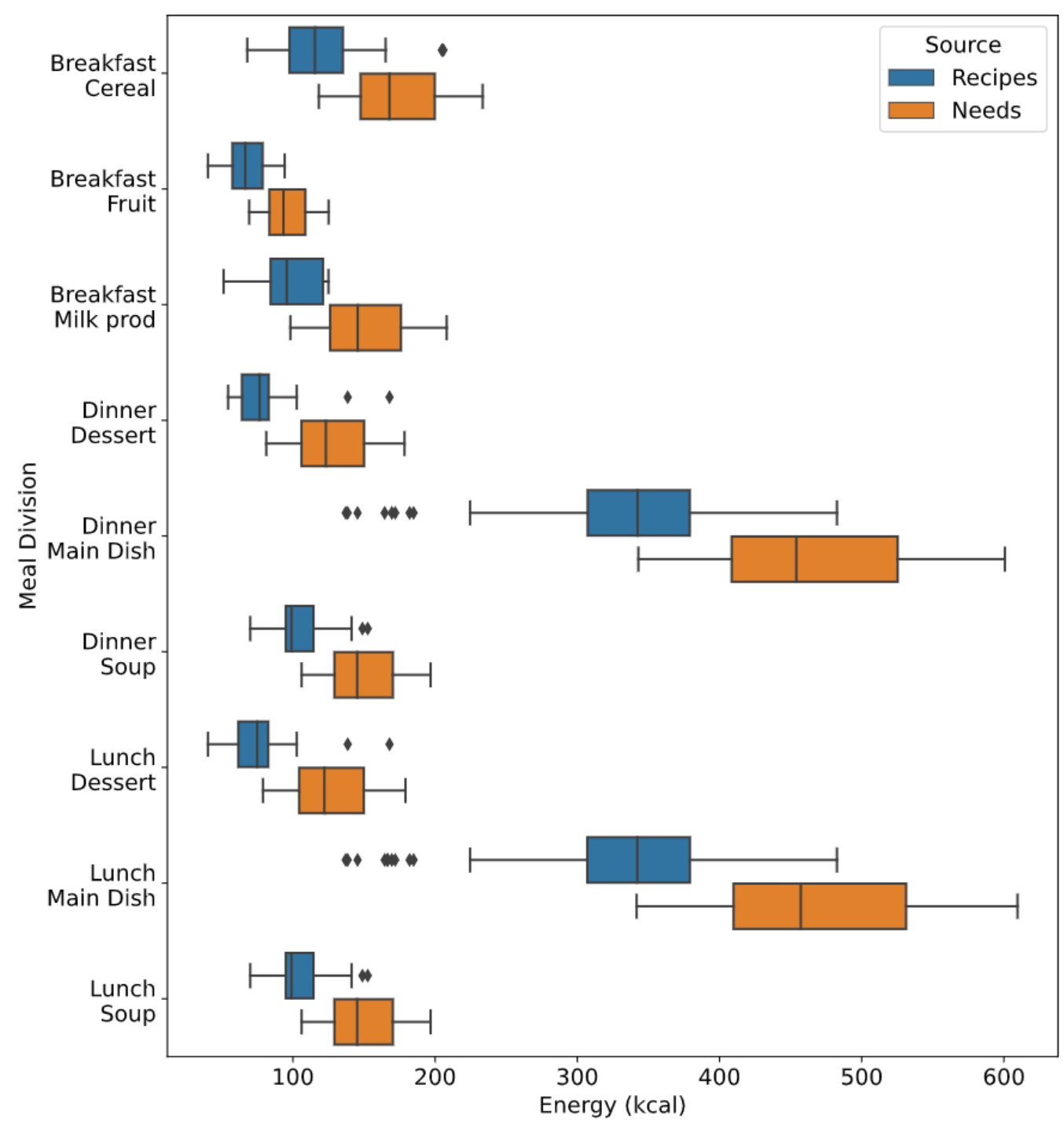

Figure 6. Box plot comparing users' energy needs with 3 selected meals and the energy content of recipes in the system for each meal division.

On the other hand, the results from the food preferences and restrictions tests suggest that food preferences are being reflected in the recommendations. Positively rated recipes are considerably more prevalent in the recommendations than negatively rated ones. Changing the weight of food preference related heuristics, or the inclusion of new ones, could also change the balance between food preferences and other criteria.

User profiles may also include restricted ingredients, recipes, and general restrictions (such as lactose intolerance). Extensive and diverse content is once more important to achieve suitable recommendations and avoid problems similar to Figure 6. No restrictions were defined in the profiles to reduce entropy in the analysis of the remaining factors.

In regards to variability in the recommendations, there is still a margin for improvement in the system by including additional heuristics as well as more and increasingly diverse recipes. Nonetheless, in Figure 4 one can see that more variability is achieved for a lower number of meals. This is because recipes are reused for different meal divisions, especially for fruit and snack dishes where recipes are more reused. This effect is illustrated in Figure 5 which shows the variability that is achieved for different meal divisions and the number of meals. Variability is higher for a lower number of meals since fewer of these meal divisions with high recipe reuse are planned. Figure 5 also shows a much higher variability for lunch and dinner's main dish than for others. This is caused by specific heuristics and restrictions targeting these specific meal divisions, since they are more important from the 
user and nutritional perspectives. It also suggests that the inclusion of additional heuristics can increase the variability of the recommendations.

The diversity of the problem formulations in the literature, as well as the lack of public datasets, make a direct quantitative comparison difficult. We have provided both absolute and normalized numbers in our results to facilitate such comparisons in the future. While other approaches are also able to create meal recommendations for multiple meals considering nutrition, preferences, and restrictions, they often use ingredients in their recommendations. The presented system uses recipes instead to meet users' expectations towards culturally acceptable and tasteful food choices in contrast to arbitrary combinations of ingredients. This also brings new challenges, especially concerning variability, as the ingredients composing each recipe need to be considered. Our study also highlights the importance of using extensive and diverse content in the development of meal recommender systems, which is often underestimated in the literature.

Moreover, previous approaches focus on supporting the management of specific diseases, whereas SousChef aims at providing daily support for their prevention. The abstraction of heuristic functions facilitates the extension of the recommender system to increase personalization considering new information about users and food. This allows its continuous improvement to increase convenience and adapt recommendations to users' context, e.g., by considering recipe's preparation time or difficulty in the recommendations to be in line with the user's context such as the available time to cook, their expertise, or ultimately helping to manage specific diseases.

Finally, the validation of other approaches is often limited to nutritional aspects, while other factors are also decisive for the acceptability of recommendations. Our validation study considered not only the nutritional suitability of the recommendations, but also food preferences and restrictions, and variability of the recommendations, which may foster its sustained use. This methodology may be replicated to validate future systems.

\section{Conclusions}

We presented SousChef, a meal recommender system capable of creating personalized meal plans for an entire week considering multiple objectives. The system uses recipes in the recommendations and may be easily extended to consider new criteria to increase personalization. It has been validated considering multiple factors related to the acceptability of the recommendations. Our results show the importance of the content used in the meal recommendations, and that it should be extensive and diverse to be able to address heterogeneous user needs. When content is suitable for the user's needs, results also show that our recommender system can create suitable recommendations considering these multiple factors. The next steps for SousChef will be to overcome the limitations shown in our tests, especially concerning the variability and content. End users will be involved to measure the acceptance of recommendations generated by the system. The topic of group recommendations [2] will be researched and applied to this domain so meal recommendations can be extended to complete families, combining the individual needs and preferences of each member.

Author Contributions: Conceptualization, D.R., J.R., T.B. and F.S.; methodology, D.R., T.B. and E.F.V.; software, D.R. and T.B.; validation, D.R., J.R., T.B. and F.S.; formal analysis, D.R.; investigation, D.R.; resources, E.F.V.; data curation, E.F.V.; writing—original draft preparation, D.R., T.B. and J.R.; writingreview and editing, F.S., E.F.V. and A.S.; visualization, D.R.; supervision, F.S.; project administration, F.S., M.S. and A.S.; funding acquisition, D.R., J.R., M.S. and A.S. All authors have read and agreed to the published version of the manuscript.

Funding: The authors would like to acknowledge the financial support obtained from the project Future Yämmi, co-funded by Compete 2020, Lisboa 2020, Portugal 2020 and the European Union, through the European Regional Development Fund (FEDER). Elsa F. Vieira (Ref CEECIND/03988/2018) thanks FCT (Fundação para a Ciência e Tecnologia) for funding through the Individual Call to Scientific Employment Stimulus, and to REQUIMTE/LAQV. 
Institutional Review Board Statement: Not applicable.

Informed Consent Statement: Not applicable.

Data Availability Statement: Not applicable.

Conflicts of Interest: The authors declare no conflict of interest.

\section{References}

1. WHO I Diet, Nutrition and the Prevention of Chronic Diseases. Available online: https://www.who.int/dietphysicalactivity/ publications/trs916/en/ (accessed on 9 December 2021).

2. Tran, T.N.T.; Felfernig, A.; Trattner, C.; Holzinger, A. Recommender Systems in the Healthcare Domain: State-of-the-Art and Research Issues. J. Intell. Inf. Syst. 2020, 57, 171-201. [CrossRef]

3. Zadeh, M.S.A.T.; Li, J.; Alian, S. Personalized Meal Planning for Diabetic Patients Using a Multi-Criteria Decision-Making Approach. In Proceedings of the 2019 IEEE International Conference on E-Health Networking, Application Services (HealthCom), Bogota, Colombia, 14-16 October 2019; pp. 1-6. [CrossRef]

4. Sapri, N.S.M.; Bedi, M.R.; Abdul-Rahman, S.; Benjamin, A.M. A Diet Recommendation for Diabetic Patients Using Integer Programming. In Proceedings of the 4th Innovation and Analytics Conference \& Exhibition Iace, Kedah, Malaysia, 25-28 March 2019; p. 040022. [CrossRef]

5. Ribeiro, D.; Machado, J.; Vasconcelos, M.J.M.; Vieira, E.; Correia de Barros, A. SousChef: Mobile Meal Recommender System for Older Adults. In Proceedings of the 3rd International Conference on Information and Communication Technologies for Ageing Well and E-Health, SCITEPRESS, Porto, Portugal, 28-29 April 2017; pp. 36-45. [CrossRef]

6. Ribeiro, D.; Ribeiro, J.; Vasconcelos, M.J.M.; Vieira, E.F.; de Barros, A.C. SousChef: Improved Meal Recommender System for Portuguese Older Adults. In Information and Communication Technologies for Ageing Well and E-Health; Röcker, C., O'Donoghue, J., Ziefle, M., Maciaszek, L., Molloy, W., Eds.; Communications in Computer and Information Science; Springer International Publishing: Cham, Switzerland, 2018; pp. 107-126. [CrossRef]

7. Ribeiro, J.; Ribeiro, D.; Schwarz, A.; Vasconcelos, M.J.M.; Gerardo, F.; van Harten, C.; Succu, R.; Davison, R.; Oliveira, T.; Silva, T.; et al. Cordon Gris: Integrated Solution for Meal Recommendations. In Proceedings of the 2018 IEEE International Conference on Pervasive Computing and Communications Workshops (PerCom Workshops), Athens, Greece, 19-23 March 2018 ; pp. 46-51.

8. Pecune, F.; Callebert, L.; Marsella, S. A Recommender System for Healthy and Personalized Recipe Recommendations. In Proceedings of the CEUR Workshop Proceedings, CEUR-WS, Online, 26 September 2020; Volume 2684, pp. 15-20.

9. Iwendi, C.; Khan, S.; Anajemba, J.H.; Bashir, A.K.; Noor, F. Realizing an Efficient IoMT-Assisted Patient Diet Recommendation System Through Machine Learning Model. IEEE Access 2020, 8, 28462-28474. [CrossRef]

10. Bushra, N.; Hasan, M. QuicklyCook: A User-Friendly Recipe Recommender. In Machine Learning and Metaheuristics Algorithms, and Applications; Thampi, S.M., Trajkovic, L., Li, K.C., Das, S., Wozniak, M., Berretti, S., Eds.; Communications in Computer and Information Science; Springer: Singapore, 2020; pp. 245-254. [CrossRef]

11. Tangseng, P.; Yamaguchi, K.; Okatani, T. Recommending Outfits from Personal Closet. In Proceedings of the 2017 IEEE International Conference on Computer Vision Workshops (ICCVW), Venice, Italy, 22-29 October 2017; pp. 2275-2279. [CrossRef]

12. Lamche, B.; Trottmann, U.; Wörndl, W. Active Learning Strategies for Exploratory Mobile Recommender Systems. In Proceedings of the 4th Workshop on Context-Awareness in Retrieval and Recommendation (CARR '14), Amsterdam, The Netherlands, 13 April 2014; Association for Computing Machinery: New York, NY, USA, 2014; pp. 10-17. [CrossRef]

13. Hung, B.T. Integrating Sentiment Analysis in Recommender Systems. In Reliability and Statistical Computing: Modeling, Methods and Applications; Pham, H., Ed.; Springer Series in Reliability Engineering; Springer International Publishing: Cham, Switzerland, 2020; pp. 127-137. [CrossRef]

14. Lamche, B.; Pollok, E.; Wörndl, W.; Groh, G. Evaluating the Effectiveness of Stereotype User Models for Recommendations on Mobile Devices. In Proceedings of the Joint Workshop on Personalized Information Access (PIA 2014), in Conjunction with the 22nd Conference on User Modeling, Adaptation and Personalization (UMAP 2014), Aalborg, Denmark, 7-11 July 2014.

15. Gao, X.; Feng, F.; He, X.; Huang, H.; Guan, X.; Feng, C.; Ming, Z.; Chua, T. Hierarchical Attention Network for Visually-Aware Food Recommendation. IEEE Trans. Multimed. 2020, 22, 1647-1659. [CrossRef]

16. Bundasak, S.; Chinnasarn, K. eMenu Recommender System Using Collaborative Filtering and Slope One Predictor. In Proceedings of the 2013 10th International Joint Conference on Computer Science and Software Engineering (JCSSE), Khon Kaen, Thailand, 29-31 May 2013; pp. 37-42. [CrossRef]

17. Yera Toledo, R.; Alzahrani, A.A.; Martínez, L. A Food Recommender System Considering Nutritional Information and User Preferences. IEEE Access 2019, 7, 96695-96711. [CrossRef]

18. Sowah, R.A.; Bampoe-Addo, A.A.; Armoo, S.K.; Saalia, F.K.; Gatsi, F.; Sarkodie-Mensah, B. Design and Development of Diabetes Management System Using Machine Learning. 2020. Available online: https://www.hindawi.com/journals/ijta/2020/8870141/ (accessed on 9 December 2021).

19. Vairale, V.S.; Shukla, S. Recommendation of Diet Using Hybrid Collaborative Filtering Learning Methods. In Advances in Computational Intelligence and Informatics; Chillarige, R.R., Distefano, S., Rawat, S.S., Eds.; Lecture Notes in Networks and Systems; Springer: Singapore, 2020; pp. 309-318. [CrossRef] 
20. Rehman, F.; Khalid, O.; Bilal, K.; Madani, S.A. Diet-Right: A Smart Food Recommendation System. KSII Trans. Internet Inf. Syst. 2017, 11, 2910-2925.

21. Shani, G.; Gunawardana, A. Evaluating Recommendation Systems. In Recommender Systems Handbook; Springer: Berlin/Heidelberg, Germany, 2011; pp. 257-297.

22. Adomavicius, G.; Tuzhilin, A. Toward the next Generation of Recommender Systems: A Survey of the State-of-the-Art and Possible Extensions. Knowl. Data Eng. IEEE Trans. 2005, 17, 734-749. [CrossRef]

23. Schröder, G.; Thiele, M.; Lehner, W. Setting Goals and Choosing Metrics for Recommender System Evaluations. In Proceedings of the UCERSTI2 Workshop at the 5th ACM Conference on Recommender Systems, Chicago, IL, USA, 23-27 October 2011; Volume 23.

24. Instituto Nacional de Saúde Dr. Ricardo Jorge. Tabela de Composição Dos Alimentos. Available online: http://portfir.insa.pt/ foodcomp/introduction (accessed on 9 December 2021).

25. Møller, A.; Ireland, J. LanguaL ${ }^{\mathrm{TM}}$ 2017-Thesaurus; Technical Report, Danish Food Informatics; EuroFIR: Brussels, Belgium, 2018. [CrossRef]

26. Lopes, C.; Torres, D.; Oliveira, A.; Severo, M.; Alarcão, V.; Guiomar, S.; Mota, J.; Teixeira, P.; Rodrigues, S.; Lobato, L.; et al. Inquérito Alimentar Nacional e de Atividade Física IAN-AF 2015-2016: Relatório Parte II; Methodological Report; University of Porto: Porto, Portugal, 2017; ISBN 978-989-746-180-4.

27. Institute of Medicine. Dietary Reference Intakes for Energy, Carbohydrate, Fiber, Fat, Fatty Acids, Cholesterol, Protein, and Amino Acids; The National Academies Press: Washington, DC, USA, 2005. [CrossRef]

28. U.S. Department of Health and Human Services and U.S. Department of Agriculture. 2015-2020 Dietary Guidelines for Americans, 8th ed.; December 2015. Available online: https://health.gov/our-work/food-nutrition/previous-dietary-guidelines/2015 (accessed on 9 December 2021). 